\title{
Evaluating thyroid nodules: predicting and selecting malignant nodules for fine-needle aspiration (FNA) cytology
}

\author{
Ravi Kumar Lingam • Mohammad Haroon Qarib • \\ Neil Samuel Tolley
}

Received: 27 January 2013 /Revised: 21 April 2013 / Accepted: 22 April 2013 /Published online: 28 May 2013

(C) The Author(s) 2013. This article is published with open access at Springerlink.com

\begin{abstract}
Objective To form and assess a set of diagnostic ultrasound criteria to select malignant nodules for fine-needle aspiration (FNA) cytology and reduce number of FNA biopsies. Methods In this prospective observational service evaluation study, 171 thyroid nodules that underwent FNA cytology were independently scored by two observers for established nodular sonographic characteristics for malignancy. The final diagnosis was confirmed by surgery or a 6month follow-up in nodules with benign cytology.

Results Logistic regression analysis and receiver operating characteristic curve analysis results indicate good and comparable predictive powers of certain ultrasound characteristics in predicting malignancy. The highest sensitivity in detecting malignancy was achieved when taking together the information of marked hypoechogenicity, microcalcification and mixed central/peripheral or central Doppler colour flow pattern. A sensitivity of $100 \%$ and a specificity of $76 \%$ were obtained in detecting malignant nodules using this criteria.
\end{abstract}

\footnotetext{
R. K. Lingam $(\bowtie) \cdot$ M. H. Qarib

Department of Radiology, Northwest London Hospitals NHS

Trust, Northwick Park and Central Middlesex Hospitals,

London, UK

e-mail: raviklingam@yahoo.co.uk

R. K. Lingam

e-mail: ravi.lingam@nhs.net

M. H. Qarib

e-mail:payman73@hotmail.com

N. S. Tolley

Department of Head and Neck Surgery, Northwest London

Hospitals NHS Trust, Northwick Park and Central Middlesex

Hospitals, London, UK

e-mail: n.tolley@imperial.ac.uk
}

Conclusions Our study proposes a set of ultrasound and colour Doppler criteria to safely select malignant thyroid nodules for FNA cytology.

Main messages:

- There is a need to safely select malignant nodules for FNA cytology and reduce unnecessary FNA

- Some ultrasound features are specific but none are independently/fully predictive of malignancy

- We have prospectively tested a set of ultrasound criteria for selecting nodules for FNA cytology

- Our ultrasound criteria detected malignant nodules with a $100 \%$ sensitivity and $76 \%$ specificity

- A high sensitivity is clinically desirable as it selects many, if not all, malignant nodules for FNA

Keywords Thyroid · Nodule $\cdot$ Malignancy $\cdot$ Ultrasound . Doppler

\section{Introduction}

In the evaluation of thyroid nodules, high-resolution ultrasound is being increasingly used to detect malignancy and guide fineneedle aspiration (FNA) for cytological analysis. There have been consensus statements and guidelines from professional bodies for the diagnostic role of ultrasound in managing thyroid nodular disease [1-6] as there is a need to safely select malignant nodules for FNA cytology and reduce unnecessary FNA or biopsies. Ultrasound features are specific for malignancy and include irregular contours, microcalcification and intranodular colour Doppler flow pattern but none of them are independently and fully predictive of malignancy [1, 2, 6-9]. A prospective study by Dominguez et al. [9] has indicated that the simultaneous presence or 
Table 1 Royal College of Pathologists (RCPath) modified British Thyroid association nomenclature and comparison to the Bethesda system for reporting thyroid cytopathology: recommended diagnostic categories (adapted from [11])

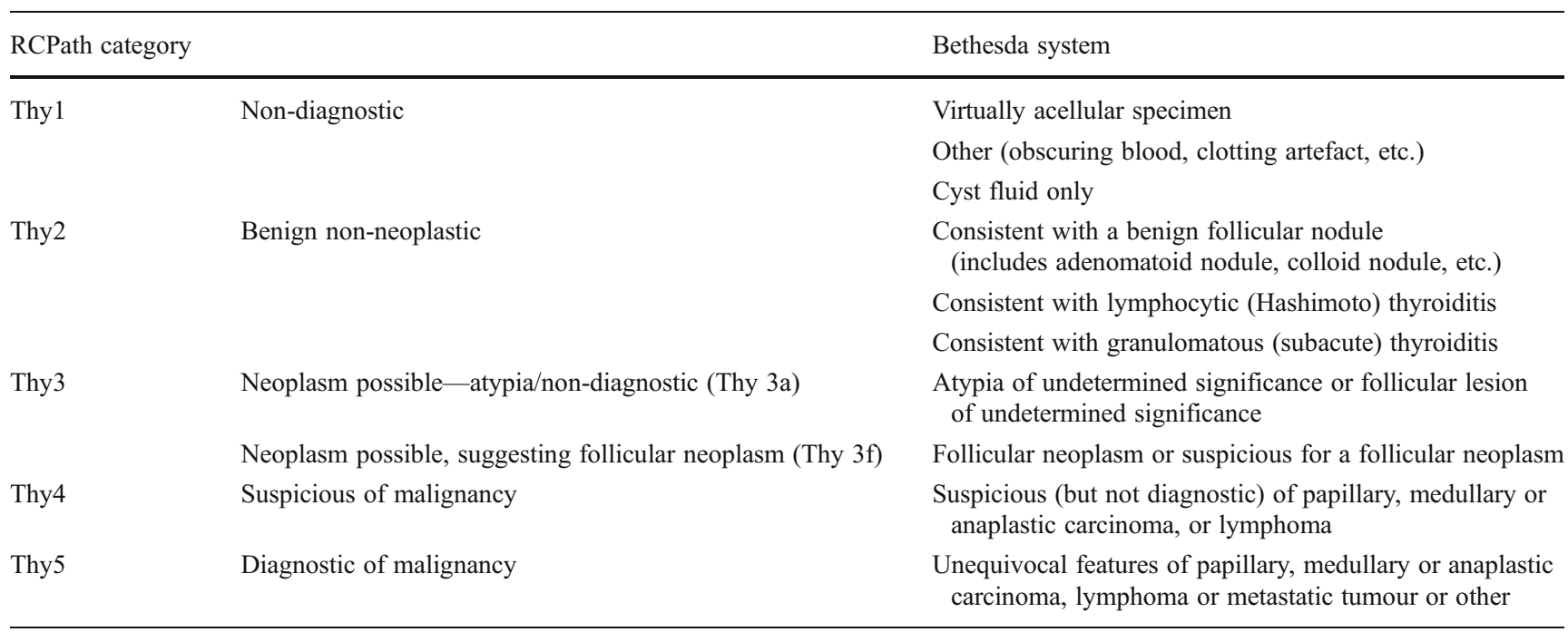

absence of these specific ultrasound and colour Doppler characteristics can change the pre-test probability for malignancy.

Currently in our institution, we offer FNA cytology analysis routinely in the evaluation of thyroid nodules but recognise the need to be discerning in selecting malignant nodules for FNA cytology and hence reduce unnecessary FNA biopsies $[6,10]$. We therefore aim to, in our prospective service analysis study, form and test the diagnostic performance of a set of ultrasound and colour Doppler criteria to safely select malignant nodules for FNA cytology and hence reduce unnecessary FNA biopsies.

\section{Materials and methods}

Ethical considerations

Ethical approval was obtained from our institution's research development unit. Written informed consent was waived by the institutional review board.

\section{Patient selection}

Our service evaluation study included a total of 215 patients seen at our head and neck ultrasound clinic from January 2006

Table 2 Logistic regression analysis for Observer 1's category scores. Observer 2's category scores are almost identical

\begin{tabular}{|c|c|c|c|c|}
\hline Outcome & Category & Malignant $n(\%)$ & Odds ratio $(95 \% \mathrm{CI})$ & $p$ value \\
\hline \multirow[t]{4}{*}{ Echogenicity } & Hyperechoic/isoechoic & $5 / 68(7 \%)$ & 1 & \\
\hline & Hypoechoic & $4 / 42(10 \%)$ & $1.33(0.33,5.24)$ & \\
\hline & Markedly hypoechoic & $8 / 13(62 \%)$ & $20.2(4.77,85.2)$ & \\
\hline & Cyst & $2 / 48(4 \%)$ & $0.55(0.10,2.95)$ & $<0.001$ \\
\hline \multirow[t]{2}{*}{ Contour } & Well defined & $11 / 157(7 \%)$ & 1 & \\
\hline & Borderline/ill-defined & $8 / 14(57 \%)$ & $17.7(5.21,60.1)$ & $<0.001$ \\
\hline \multirow[t]{3}{*}{ Colour flow } & No flow/peripheral flow & $0 / 78(0 \%)$ & & \\
\hline & Peripheral $>$ central flow & $5 / 43(12 \%)$ & & \\
\hline & Central flow $\geq$ peripheral flow & $14 / 50(28 \%)$ & a & $<0.001$ \\
\hline \multirow[t]{2}{*}{ Colour flow (grouped) } & No flow or peripheral $>$ central flow & $5 / 121(7 \%)$ & 1 & \\
\hline & Central flow $\geq$ peripheral flow & $14 / 50(46 \%)$ & $9.02(3.04,26.8)$ & $<0.001$ \\
\hline \multirow[t]{3}{*}{ Calcification } & No & $13 / 157(8 \%)$ & & \\
\hline & Coarse & $1 / 9(11 \%)$ & & \\
\hline & Microcalcification & $5 / 5(100 \%)$ & a & $<0.001$ \\
\hline \multirow[t]{2}{*}{ Calcification (grouped) } & No & $13 / 157(8 \%)$ & 1 & \\
\hline & Coarse or microcalcification & $6 / 14(43 \%)$ & $6.63(2.61,16.8)$ & 0.002 \\
\hline
\end{tabular}

${ }^{a}$ Unable to calculate odds ratios or perform logistic regression due to all values in some categories having said outcome. Analysis using Fisher's exact test 
Table 3 The results of the multivariable analysis based on $\mathrm{Ob}-$ server 1's category scores analysis indicated some evidence that all four factors were significantly associated with the outcome (Model 1). However, the result for contour was not quite statistically significant. When only strictly significant variables were considered (Model 2), the results suggested that echogenicity, colour flow and calcification were still all significantly associated with the outcome. Observer 2's category scores are almost identical

\begin{tabular}{|c|c|c|c|}
\hline Outcome & Category & Odds ratio $(95 \% \mathrm{CI})$ & $p$ value \\
\hline \multicolumn{4}{|l|}{ Model 1} \\
\hline \multirow[t]{4}{*}{ Echogenicity } & Hyperechoic/isoechoic & 1 & \\
\hline & Hypoechoic & $0.55(0.08,3.56)$ & \\
\hline & Markedly hypoechoic & $6.81(1.11,41.9)$ & \\
\hline & Cyst & $0.48(0.06,3.81)$ & 0.04 \\
\hline \multirow[t]{2}{*}{ Contour } & Well defined & 1 & \\
\hline & Borderline/ill-defined & $8.11(0.72,91.2)$ & 0.09 \\
\hline \multirow[t]{2}{*}{ Colour flow } & No flow or peripheral $>$ central flow & 1 & \\
\hline & Central flow $\geq$ peripheral flow & $26.0(4.07,166)$ & 0.001 \\
\hline \multirow[t]{2}{*}{ Calcification } & No calcification & 1 & \\
\hline & Coarse calcification or microcalcification & $5.83(1.18,28.9)$ & 0.03 \\
\hline \multicolumn{4}{|l|}{ Model 2} \\
\hline \multirow[t]{4}{*}{ Echogenicity } & Hyperechoic/isoechoic & 1 & \\
\hline & Hypoechoic & $0.84(0.16,4.33)$ & \\
\hline & Markedly hypoechoic & $9.69(1.81,51.9)$ & \\
\hline & Cyst & $0.42(0.05,3.67)$ & 0.01 \\
\hline \multirow[t]{2}{*}{ Colour flow } & No flow or peripheral $>$ central flow & 1 & \\
\hline & Central flow $\geq$ peripheral flow & $25.1(4.05,156)$ & 0.001 \\
\hline \multirow[t]{2}{*}{ Calcification } & No & 1 & \\
\hline & Coarse calcification or Microcalcification & $12.1(2.66,54.9)$ & 0.001 \\
\hline
\end{tabular}

to July 2010. They were referred for ultrasound of the thyroid gland and FNA cytology by general practitioners and hospital clinicians. The clinical indications included lump in the neck and assessment of goitre.

\section{Ultrasound and FNA assessment}

All the patients had ultrasound examination and FNA cytology performed in the clinic by a consultant radiologist with 4 years' experience in thyroid ultrasound at the time of the study, in accordance with the requirement of our existing service provision. Ultrasound with colour Doppler was performed with an HDI 5000 scanner (Advanced Technology Laboratories, Bothell, WA) using a 12-MHz linear probe from 2006 to 2007 and an Acuson Antares scanner (Siemens Medical Solutions, USA) with a 13.5-MHz linear probe from 2007 to 2008. Colour Doppler ultrasonography was used for the assessment of nodule vascularity for all nodules on longitudinal and transverse planes. The amplifier gain was individually established in each stud$\mathrm{y}$; the amplifier gain was raised until random colour noise appeared and then slightly lowered to situate it at a level immediately under the point of appearance of random colour noise.

In line with our existing service provision, all patients with nodules were selected by the radiologist for FNA cytology analysis. All solitary nodules were selected for FNA cytology regardless of their sonographic and colour Doppler features. When a multinodular thyroid gland is encountered, the largest nodule was included in the study unless an atypical nodule with ultrasound features specific for malignancy was encountered $[2,6]$. Recognised features suspicious for malignancy used include: microcalcification, ill-defined contours, marked hypogenicity and presence of internal or mixed vascularity $[1,2]$. FNA was performed using a 21-G needle following verbal consent and smeared using an air-dried and fixed slide. FNA of a mixed solidcystic nodule was directed at the solid component. If abnormal regional lymphadenopathy was present, FNA was also performed at the abnormal lymph nodes.

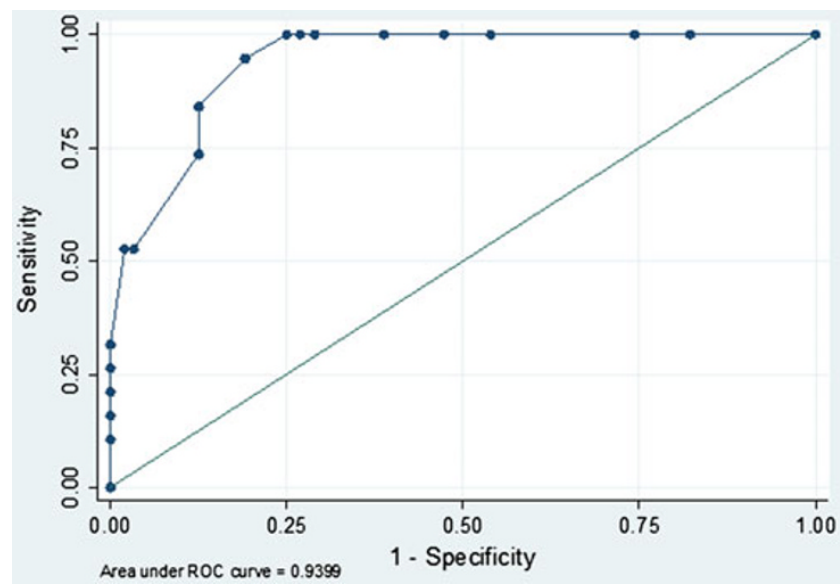

Fig. 1 Receiver operating characteristic (ROC) curve for observer 1 shows high area-under-the-curve (AUC) values, indicating good diagnostic performance in predicting the outcome. The ROC curve for observer 2 is similar 
Table 4 A summary of the performance of two ROC curve cutoffs that could be best used to predict the final diagnosis. One cut-off is chosen to give the best sensitivity and a second to give the optimum combination of sensitivity and specificity (overall accuracy)

\begin{tabular}{lll}
\hline Method & Statistic & Estimate $(95 \% \mathrm{CI})$ \\
\hline $\begin{array}{l}\text { Regression results } 1 \\
\text { (best sensitivity) }\end{array}$ & Sensitivity & $1.00(0.82,1.00)$ \\
& Specificity & $0.75(0.67,0.82)$ \\
& Positive predictive value & $0.33(0.21,0.47)$ \\
& Negative predictive value & $1.00(0.96,1.00)$ \\
Regression results 2 & Overall accuracy & $0.78(0.71,0.84)$ \\
(best combination of & Sensitivity & $0.95(0.74,1.00)$ \\
sensitivity & Specificity & $0.81(0.74,0.87)$ \\
and specificity) & Positive predictive value & $0.38(0.25,0.54)$ \\
& Negative predictive value & $0.99(0.96,1.00)$ \\
& Overall accuracy & $0.82(0.76,0.88)$ \\
\hline
\end{tabular}

\section{Ultrasound and colour Doppler scoring}

For the purpose of the study, the sonographic and colour Doppler features of the nodule that was selected for FNA cytology analysis were categorised for echogenicity, contours, nodular colour Doppler flow characteristics and the presence of calcification, all of which are regarded as important ultrasound signs associated with malignancy $[1,2]$. The echogenicity of the nodule was compared with the surrounding parenchyma and was classified as markedly hypoechoic, hypoechoic, isoechoic/hyperechoic or cystic. If a cystic nodule is encountered, it is assessed for the other characteristics as with the solid nodules, such as contour, internal vascularity and calcification in its soft tissue component. Markedly hypoechoic was defined as lower echogenicity than the adjacent strap muscles. Contours were scored as well defined (crisp) margins or ill-defined (blurred, infiltrative) margins. The presence of calcification was scored as coarse calcification or microcalcifications, the latter being defined as tiny (less than $2 \mathrm{~mm}$ ) linear hyperechoic foci with or without acoustic shadow. Nodular colour Doppler flow pattern was categorised as no flow, peripheral flow only, mixed flow (peripheral more than central), mixed flow (central more than peripheral) and central flow only. To evaluate for interobserver agreement, the nodules were also scanned and scored independently in the clinic by a second observer, a sonographer with 3 years' experience in thyroid ultrasound.

\section{Cytological assessment and final diagnosis}

As per our existing service provision, the slides were examined by cytologists experienced in thyroid cytology and graded in line with the British Thyroid Association [3] and Royal College of Pathologists [11] guidelines (Table 1). All nodules with a Thy 2 cytology diagnosis denote benign nonneoplastic lesions, typically colloid nodules, thyroiditis and hyperplastic nodules $[3,11]$. Occasional hyperplastic nodules show a microfollicular pattern with minimal colloid precluding a Thy2 diagnosis as it is not possible to differentiate from a neoplastic or potential malignant lesion. These nodules are classified as Thy 3 [3, 4]. All nodules with a potential malignant cytology grading (Thy3, Thy 4 and Thy5), were referred for surgery, with histological diagnosis obtained in all these cases.

The final diagnosis of malignant nodules is based on surgery with histological diagnosis. The diagnosis of benign nonneoplastic nodules was based on Thy 2 cytology and they were also followed-up after 6 months to ensure stability; any nodule with a $20 \%$ increase in diameter with minimum increase in two or more dimensions of $2 \mathrm{~mm}$ would be subjected to a further FNA cytological analysis to establish benignity [4]. Surgery with histological confirmation was available for some benign nodules where surgery was performed for cosmetic or compressive symptoms or nodules with Thy 3 cytology.

\section{Statistical analyses}

Logistic regression was used to predict the probability of a malignant nodule for each patient based on the four ultrasound characteristics (echogenicity, contour, colour flow, presence of calcification). Receiver operating characteristic (ROC) curve analysis was used to examine the performance of these predicted values in determining a malignant result. ROC curve analysis was performed using the Stata software (version 9.2; StataCorp LP, Texas, USA).

Table 5 Proposed set of ultrasound criteria for selecting thyroid nodules for FNA cytology analysis. Nodules are selected if any of these features are seen on ultrasound

Thyroid nodular ultrasound features for selecting nodules for FNA

Central or mixed colour Doppler flow pattern

Markedly hypoechoic

Microcalcification 
Table 6 Diagnostic performance of the two observers in detecting malignant thyroid nodules using ultrasound criteria outlined in Table 4. This illustrates a safe approach where all malignant nodules are selected for FNA cytology analysis

\begin{tabular}{lll}
\hline & Observer $1(95 \% \mathrm{CI})$ & Observer 2 $(95 \% \mathrm{CI})$ \\
\hline Sensitivity & $1.00(0.78,1.00)$ & $1.00(0.78,1.00)$ \\
Specificity & $0.76(0.69,0.83)$ & $0.72(0.64,0.79)$ \\
Positive predictive value & $0.33(0.21,0.48)$ & $0.30(0.19,0.43)$ \\
Negative predictive value & $1.00(0.96,1.00)$ & $1.00(0.96,1.00)$ \\
\hline
\end{tabular}

\section{Results}

Forty-four patients were excluded from the study as they were lost to follow-up scans to ensure benignity or the histology reports were unavailable (surgery at another centre or surgery refused). Of the 171 cases that were included in the study, there were 34 male and 141 female patients, and the median age was 48 years (range 18-88 years). One hundred and fifty-three were benign (140 were non-neoplastic and 13 were benign neoplasms [10 follicular adenoma, 3 hurthle cell neoplasm on histology]) and 18 were malignant (10 papillary carcinoma, 5 follicular carcinoma, 1 hurtle cell microcarcinoma, 1 medullary carcinoma and 1 lymphoma on histology). All the malignant nodules had cytology scores of Thy 3 , Thy 4 or Thy 5, apart from one case of follicular carcinoma with a Thy1 score. Of the 140 benign non-neoplastic nodules, 10 cases had an indeterminate Thy 3 cytology grade and therefore underwent surgery with histological confirmation of absence of cancer. The remaining 130 cases had Thy 2 cytology grade, 12 of which had surgery and histological confirmation of benignity.

The thyroid nodules vary in size, with diameters ranging from $0.6 \mathrm{~cm}$ to $7.5 \mathrm{~cm}$. There is no significant difference in size between the benign nodules (mean $2.7 \mathrm{~cm}$, SD $1.7 \mathrm{~cm}$ ) and the malignant nodules (mean $2.8 \mathrm{~cm}$, SD $1.1 \mathrm{~cm}$ ) (unpaired $t$-test, $p=0.68$ ). There was also no significant difference between benign and malignant nodules in being palpable or not palpable (chi-squared test $p=0.15$ ) or in being solitary or occurring in a multinodular gland (chi-squared test $p=0.22$ ).

Logistic regression analysis results for both observers (Table 2) suggest that all four ultrasound characteristics (echogenicity, contour, colour flow, presence of calcification) are significantly associated with the final diagnosis. Marked hypoechogenicity, ill defined contour, mixed central/peripheral flow or central flow and calcification are features most likely to be associated with malignant nodules. The results of the multivariable analysis for both observers (Table 3 ) indicate that echogenicity, calcification and colour flow are still all significantly associated with the final diagnosis. However, the result for contour was not quite statistically significant. All the five nodules demonstrating microcalcification were malignant.

The area under the ROC curves (Fig. 1) for both observers are of high value indicating good and comparable predictive powers of the logistic regression analysis in predicting the final diagnosis. The area under the curve (AUC) value for observer 1 and 2 is identical at 0.94 with $95 \%$ CI of 0.90-0.98. Two different cut-offs were chosen at the ROC curves, one to give the best sensitivity, and a second to give the optimum combination of sensitivity and specificity (overall accuracy). The results are identical for both observers and are shown in Table 4.

Based on the results of the logistic regression and ROC curve analysis and the fact that all cases demonstrating microcalcification were malignant, we propose a set of ultrasound selection criteria for detecting malignant nodules and selecting them for FNA cytology (Table 5). The diagnostic performance of this selection criteria is listed in Table 6.

\section{Discussion}

In the investigation of thyroid nodules, there is a need to safely select nodules for FNA cytological analysis to maximise benefits and minimise cost, given the high incidence of thyroid nodules [6,10]. Evidence is gathering that the malignant potential of thyroid nodules may not be strongly associated with nodule size or palpability or whether they are solitary or occur in a multinodular gland [1, 2, 5-8, 10]. Clinical risk factors such as a history of head and neck
Fig. 2 Ultrasound images of the thyroid gland showing (a) markedly hypoechoic nodule (between callipers) with illdefined irregular contour and (b) mixed flow pattern with central flow on colour Doppler. Surgical excision and histology revealed a papillary thyroid carcinoma
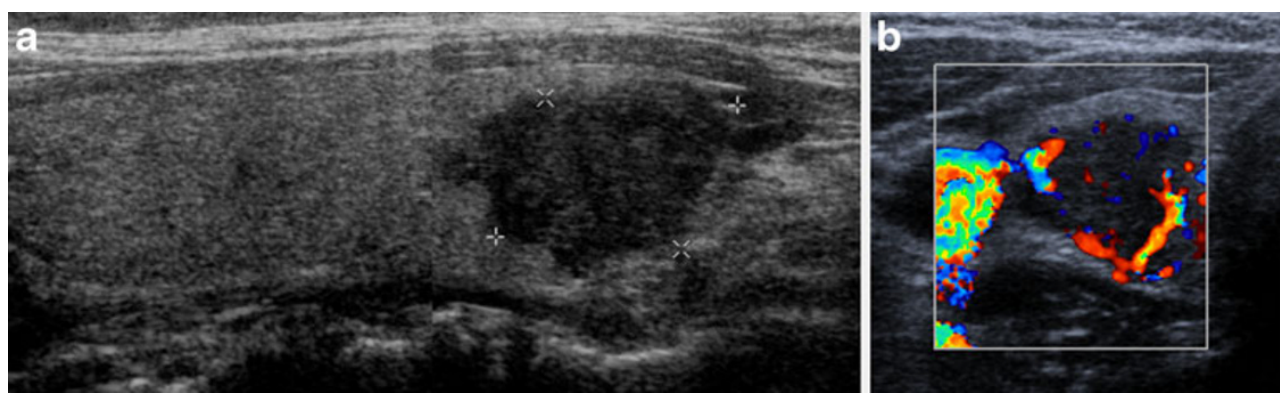


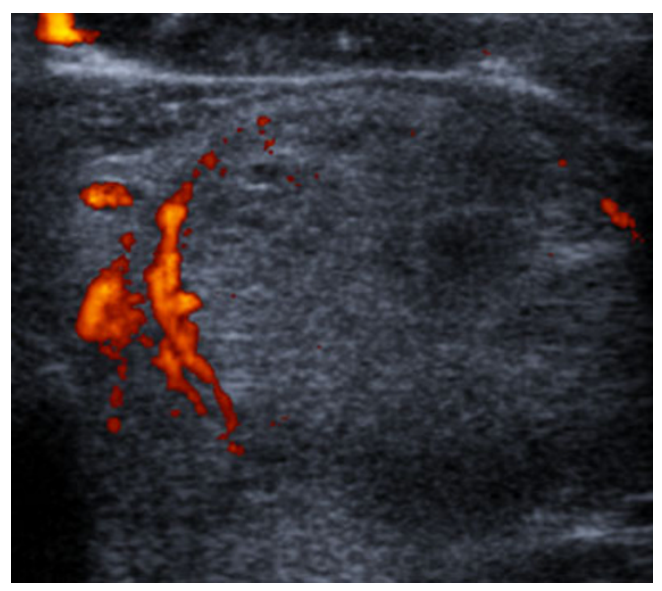

Fig. 3 Ultrasound of the thyroid gland showing a benign colloid nodule which has typical ultrasound features of a non-neoplastic nodule. This nodule has a well-defined contour, an isoechoic/hyperechoic echo texture and demonstrates peripheral flow on colour Doppler

irradiation, thyroid cancer, a family history of thyroid cancer or familial adenomatous polyposis, rapid growth, vocal cord paralysis and regional lymphadenopathy $[1,6,8]$ are uncommon. There is thus increasing reliance on ultrasound and colour Doppler, by virtue of certain specific characteristics for detecting malignancy, to select nodules for FNA or biopsy [2]. It has also been recommended that in a multinodular gland FNA should be targeted by suspicious ultrasound features rather than by a nodule being clinically dominant $[2,5,6]$.

In our service evaluation study, we have tested a set of ultrasound and colour Doppler selection criteria for detecting malignant nodules and selecting them for FNA cytology (Table 5). The characteristics of calcification, mixed colour flow pattern and markedly hypoechoic echo texture (Fig. 2) all have high odds ratios on the logistic regression (multivariable) analysis. In particular, microcalcification is the most specific sign, with all the five nodules demonstrating it being malignant. A sensitivity of $100 \%$ (Table 6 ) is obtained making it a safe approach with all malignant nodules 'earmarked' and selected for FNA cytology. Even though this selection criterion has a lower specificity of $76 \%$, we have demonstrated that it can reduce substantially the number of required FNA biopsies. In our series of 171 patients, 117 out of the 153 benign nodules (Fig. 3) would not have required FNA cytology analysis to establish benignity if the selection criterion (Table 5) was applied in the first instance, with consequent benefit to both patient and service provision and reduction in cost.

The lower specificity is due to 36 false-positive cases. The most common nodular characteristic leading to false positive diagnosis is the presence of central or mixed colour flow pattern on Doppler ultrasound seen in 32 out of 36 falsepositive cases (Fig. 4). But it is this characteristic that is the only abnormal characteristic in 6 of the 18 malignant nodules. The high sensitivity and lower positive predictive value of colour Doppler pattern in detecting malignancy is well documented in other studies $[7,12,13]$. A less common cause of false-positive result is marked hypoechogenicity (five cases). It is, however, the only abnormal feature in two malignant nodules. Seven out of eight cases of coarse calcification were false-positive for malignancy and in the one case of malignancy with coarse calcification, florid intranodular colour flow was also seen. By contrast, all five cases of microcalcification were malignant nodules, making it the most specific sign for malignancy. This sign is, however, also associated with either marked hypoechogenicity or marked intranodular colour flow in four out of five cases. Of a particular note is one case of histologically proven follicular carcinoma which had two nondiagnostic Thy 1 cytology results preoperatively. This nodule was markedly hypoechoic and had marked central vascularity on ultrasound. Hence, in the case of a nodule where cytology is repeatedly non-diagnostic, ultrasound with colour Doppler can be valuable in helping the surgeon decide on surgery.

With regards to prospective studies investigating the use of ultrasound with colour Doppler [7, 9, 14, 15], our study is, to our knowledge, the larger of two prospective studies [14] testing the diagnostic performance of a set of ultrasound and colour Doppler criteria in detecting malignancy in palpable and non-palpable thyroid nodules, where the
Fig. 4 False-positive cases for malignancy. Ultrasound images of (a) a solid - cystic follicular adenoma and (b) a benign hyperplastic nodule. The nodules demonstrated mixed (central and peripheral) colour flow pattern on colour Doppler and selected for FNA using our criteria in Table 5
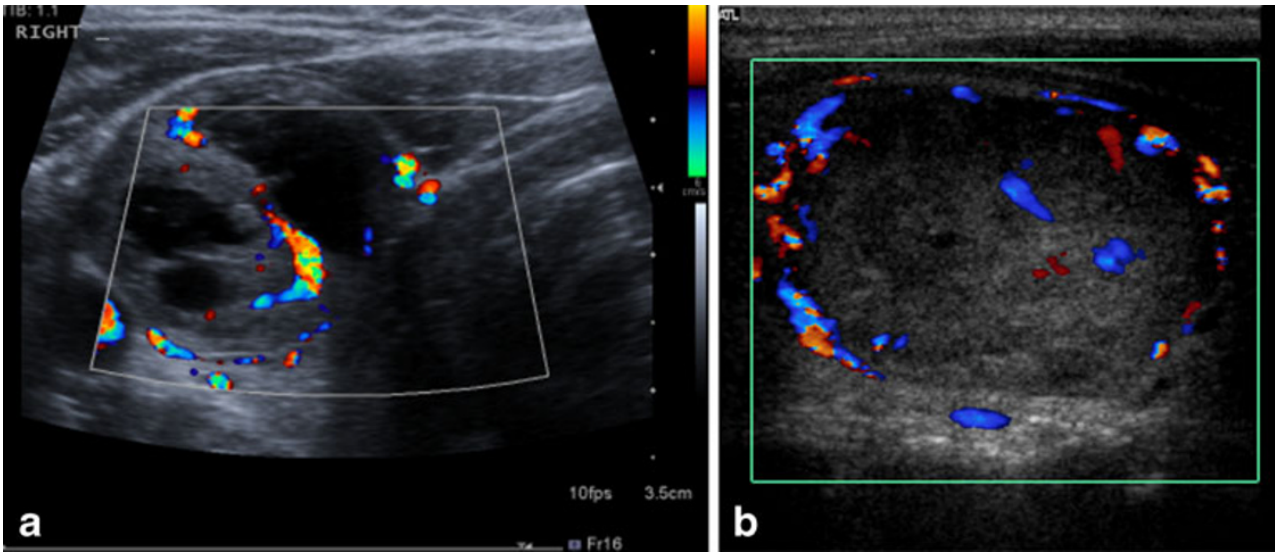
final diagnosis is established by histology for all malignant and indeterminate nodules and cytology with follow-up for all nodules with benign cytology. In a smaller study of 66 patients (78 thyroid nodules), Yunus et al. [14] demonstrated a slightly lower sensitivity and specificity of $93.8 \%$ and $66 \%$ respectively compared with ours in detecting malignancy for a set of selection criteria that are similar to ours but also included a taller than wide shape of the nodule and nodular irregularity/microlobulations. Their study, however, included only solid nodules (cystic nodules excluded).

In interpreting the results, it is important to note the limitations of our prospective study. Our study is part of a service evaluation with the aim of informing the evidence around rationalising selection for FNA. Many patients in our hospital catchment area are managed in primary care by general practitioners who refer mainly more complex cases to our clinic. In addition, there is selection bias with many nodules, with benign cytology being excluded from the study, having not being followed-up as part of the criteria to establish benignity. Bias is also introduced as nodules are not randomly selected for FNA in a multinodular gland but selected by size or atypical ultrasound features. Having said this, $10 \%$ of the cases in our sample were malignant and comparable with an overall incidence of $9.2-13 \%$ in patients with thyroid nodules selected for FNA [1]. Even though our sample size is smaller than the few other prospective studies $[7,9,15]$ evaluating thyroid ultrasound characteristics with colour Doppler, it is the largest prospective study to date investigating the use of a set of ultrasound and colour Doppler criteria in selecting malignancy nodules for FNA or biopsy. As with many studies in the literature evaluating thyroid ultrasound characteristics, the diagnosis of a benign nodule in the majority of our patients is a cytological rather than a histological one, as these patients do not routinely undergo surgery for benign non-neoplastic nodules. We hope to offset the limitations of cytological diagnosis by performing FNA under ultrasound guidance by an experienced radiologist rather than blindly $[16,17]$ and with slides read by dedicated head and neck cytologists. By additionally following-up these nodules for 6 months or more to ensure stability in size [4], we hope to support the benign nature of these nodules. None of the Thy 2 nodules in our study were later classified as neoplastic on follow-up. However, we note from the observation of Ito et al. [18] that papillary microcarcinomas may not increase in size over 1or 2-year follow-up. It may be worthwhile to note that if the study was performed in a dedicated head and neck ultrasound clinic (ultrasound being an operator-dependent procedure), the results may not be replicated by observers in a general clinic. Finally, our study uses the same dataset to determine the set of ultrasound selection criteria for detecting malignancy nodules as well as its accuracy. This requires validation by an independent dataset, preferably in the form of a larger multicentre study.

\section{Conclusion}

Given the limitations of our study, it adds to the growing body of evidence purporting the use of ultrasound and colour Doppler as a safe, reproducible and pragmatic approach for investigating thyroid nodules for the presence of malignancy and reducing unnecessary FNA biopsies.

\section{Conflict of interest None}

Open Access This article is distributed under the terms of the Creative Commons Attribution License which permits any use, distribution, and reproduction in any medium, provided the original author(s) and the source are credited.

\section{References}

1. Frates MC, Benson CB, Charboneau JW, Cibas ES, Clark OH, Coleman BG et al (2006) Management of thyroid nodules detected at US: society of radiologists in ultrasound consensus conference statement. Ultrasound Q 22:231-238

2. Gharib H, Papini E, Valcavi R, Baskin HJ, Crescenzi A, Dottorini $\mathrm{ME}$ et al (2006) American association of clinical endocrinologists and associazione medici endocrinologi medical guidelines for clinical practice for the diagnosis and management of thyroid nodules. Endocr Pract 12:63-102

3. Perros P (2007) British thyroid association. Guidelines for the management of thyroid cancer, 2nd edn. Royal College of Physicians, London

4. Cooper DS, Doherty GM, Haugen BR, Kloos RT, Lee SL, Mandel SJ et al (2006) Management guidelines for patients with thyroid nodules and differentiated thyroid cancer. Thyroid 16:109-142

5. Gharib H, Papini E, Paschke R, Duick DS, Valcavi R, Hegedus L et al (2010) American association of clinical endocrinologists, Associazione Medici Endocrinologi, and European Thyroid Association medical guidelines for clinical practice for the diagnosis and management of thyroid nodules: executive summary of recommendations. J Endocrinol Invest $33(5$ Suppl):51-56

6. Cooper DS, Doherty GM, Haugen BR, Kloos RT, Lee SL, Mandel SJ et al (2009) Revised American thyroid association management guidelines for patients with thyroid nodules and differentiated thyroid cancer. Thyroid 19:1167-1214

7. Papini E, Guglielmi R, Bianchini A, Crescenzi A, Taccogna S, Nardi $F$ et al (2002) Risk of malignancy in nonpalpable thyroid nodules: predictive value of ultrasound and color-Doppler features. J Clin Endocrinol Metab 87:1941-1946

8. Hatton R, Patel M, Devendra D (2009) Thyroid swellings. BMJ 339:b2563

9. Dominguez JM, Baudrand R, Cerda J, Campusano C, Fardella C, Arteaga E et al (2011) An ultrasound model to discriminate the risk of thyroid carcinoma. Acad Radiol 18:242-245

10. Ahn SS, Kim EK, Kang DR, Lim SK, Kwak JY, Kim MJ (2010) Biopsy of thyroid nodules: comparison of three sets of guidelines. AJR Am J Roentgenol 194:31-37

11. Cross P, Chandra A, Giles T, Johnson S, Kocjan G, Poller D et al (2009) Royal college of pathologists. Guidance on the reporting of thyroid cytology specimens. The Royal College of Pathologists, London 
12. Rago T, Vitti P, Chiovato L, Mazzeo S, De Liperi A, Miccoli P et al (1998) Role of conventional ultrasonography and colour flow Doppler sonography in predicying malignancy in "cold" thyroid nodules. Eur J Endocrinol 138:41-46

13. Appetecchia M, Solivetti FM (2006) The association of colour flow Doppler sonography and conventional ultrasonography improves the diagnosis of thyroid carcinoma. Horm Res 66:249-256

14. Yunus M, Ahmed Z (2010) Significance of ultrasound features in predicting malignant solid thyroid nodules: need for fine-needle aspiration. J Pak Med Assoc 60:848-853

15. Stacul F, Bertolotto M, De Gobbis F, Calderan L, Cioffi V, Romano A et al (2007) US, colour-Doppler US and fine-needle aspiration biopsy in the diagnosis of thyroid nodules. Radiol Med 112:751-762

16. Carmeci C, Jeffrey RB, McDougall IR, Nowels KW, Weigel RJ (1998) Ultrasound-guided fine-needle aspiration biopsy of thyroid masses. Thyroid 8:283-289

17. Danese D, Sciacchitano S, Farsetti A, Andreoli M, Pontecorvi A (1998) Diagnostic accuracy of conventional versus sonographyguided fine-needle aspiration biopsy of thyroid nodules. Thyroid $8: 15-21$

18. Ito Y, Uruno T, Nakano K, Takamura Y, Miya A, Kobayashi K et al (2003) An observation trial without surgical treatment in patients with papillary microcarcinoma of the thyroid. Thyroid 3:381-387 